\title{
MAPS WHICH PRESERVE GRAPHS
}

\author{
VAN C. NALL
}

(Communicated by Doug W. Curtis)

\begin{abstract}
In 1976 Eberhart, Fugate, and Gordh proved that the weakly confluent image of a graph is a graph. A much weaker condition on the map is introduced called partial confluence, and it is shown that the image of a graph is a graph if and only if the map is partially confluent.

In addition, it is shown that certain properties of one-dimensional continua are preserved by partially confluent maps, generalizing theorems of Cook and Lelek, Tymchatyn and Lelek, and Grace and Vought. Also, some continua in addition to graphs are shown to be the images of partially confluent maps only.
\end{abstract}

A compactum is a compact metric space, and a continuum is a connected campactum. A map is a continuous function. A map $f$ from a compactum $X$ onto a compactum $Y$ is called (1) confluent if for each continuum $K$ in $Y$, each component of $f^{-1}(K)$ maps onto $K$, (2) weakly confluent if for each continuum $K$ in $Y$, some component of $f^{-1}(K)$ maps onto $K,(3)$ pseudoconfluent if for each irreducible continuum $K$ in $Y$, some component of $f^{-1}(K)$ maps onto $K,(4)$ partially confluent if each continuum $K$ in $Y$ is the union of a finite number of continua which are the images of components of $f^{-1}(K)$, and (5) $n$-partially confluent for some positive integer $n$ if each continuum $K$ in $Y$ is the union of $n$ continua which are the images of components of $f^{-1}(K)$. The following implications hold.

$$
\text { confluence } \Rightarrow \text { weak confluence } \begin{gathered}
\text { pseudoconfluence } \\
\Downarrow \text { partial confluence }
\end{gathered}
$$

I. Examples. Since many of the results in this paper for partially confluent maps are similar to those obtained in [2 and 6] for pseudoconfluent maps, it is important to illustrate the difference between these two generalizations of weak confluence.

I.1. EXAMPLE. A partially confluent and pseudoconfluent map onto a triod.

Figure 1 illustrates a map from an arc onto a triod. Simply project the arc as indicated onto the triod to produce a map which is partially confluent (in fact, 2-partially confluent) and pseudoconfluent but not weakly confluent.

Received by the editors June 13, 1985 and, in revised form, August 2, 1985 and August 11, 1986.

1980 Mathematics Subject Classification (1985 Revision). Primary 54F20; Secondary 54F55.

Key words and phrases. Continuum, weakly confluent, partially confluent, suslinean, graph.

This research was supported in part by a grant from the University of Richmond Research Committee. 


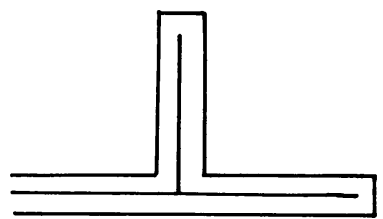

FIGURE 1

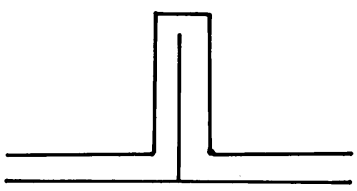

FIGURE 2

I.2. EXAMPLE. A partially confluent map which is not pseudoconfluent.

I.3. EXAMPLE. A pseudoconfluent map which is not partially confluent. For each positive integer $i$ let

$$
L_{i}=\{(r, \theta) \mid r \in[0,1] \text { and } \theta=\pi / 2 i\}
$$

and

$$
L=\{(r, \theta) \mid r \in[0,1] \text { and } \theta=0\} .
$$

Let $F$ be the closure of the union of the $L_{i}$ 's. The fan $F$ will be the range space.

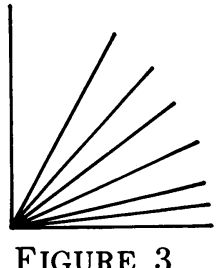

To form the domain, for each $i$ let $X_{i}$ be the closure of the union of the set of straight lines joining the following sequence of points:

$$
\left(\frac{i-1}{2^{j}}, 0\right),\left(\frac{i-1}{2^{j}}+\frac{1}{2^{j+1}}, 1\right),\left(\frac{i-1}{2^{j}}+\frac{2}{2^{j+2}},-1\right),\left(\frac{i-1}{2^{i}}+\frac{3}{2^{i+3}}, 1\right), \ldots
$$

Each $X_{i}$ is homeomorphic to the graph of $\sin (1 / x)$ together with the limit bar. Let $X$ represent the union of the $X_{i}$ 's.

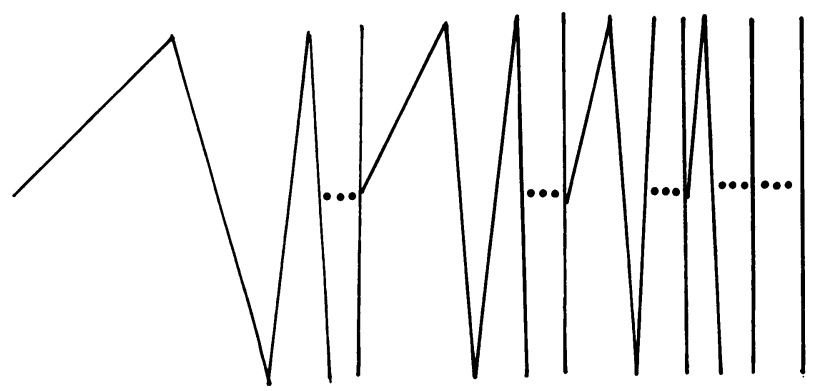

FIGURE 4 
For each $i$ map $X_{i}$ into $F$ as follows: project each component of

$$
\left\{(x, y) \in X_{i} \mid y \geq 0\right\}
$$

onto the $y$-axis and then rotate it onto $L_{i}$. The components of

$$
\left\{(x, y) \in X_{i} \mid y \leq 0\right\}
$$

are taken in sequence from left to right and mapped in a similar fashion onto $L_{i+1}, L_{i+2}, L_{i+3}, \ldots$ respectively. Extend this map continuously to include the limit line at $x=1$ and the result is a pseudoconfluent map from $X$ onto $F$. The subcontinuum

$$
\{(r, \theta) \mid r \in[0,1 / 2] \text { and } \theta=0 \text { or } \theta=1 / n \text { for some } n=1,2,3, \ldots\}
$$

of $F$ is not the finite union of images of continua from $X$.

II. Partially confluent images of one-dimensional continua. A compactum $X$ is suslinean if every pairwise disjoint collection of nondegenerate subcontinua of $X$ is finite or countable. A compactum $X$ is finitely suslinean if every pairwise disjoint collection of subcontinua of $X$ having diameter greater than a positive number is finite. A compactum $X$ is regular if at each point of $X$ there is a local basis consisting of open sets with finite boundaries.

II.1. THEOREM. If $f$ is a partially confluent map from a suslinean compactum $X$ onto a compactum $Y$, then $Y$ is suslinean.

PROOF. Let $\left\{K_{\lambda}\right\}_{\lambda \in \Lambda}$ be a pairwise disjoint collection of subcontinua of $Y$. For each $\lambda$ in $\Lambda$ the partial confluence of $f$ guarantees the existence of a nondegenerate continuum $C_{\lambda}$ in $X$ which maps into $K_{\lambda}$. Since the $C_{\lambda}$ 's form a pairwise disjoint collection in $X, \Lambda$ must be countable.

The following characterization of regular continua is due to Lelek.

A continuum $X$ is regular if and only if for each number $\varepsilon>0$ there exists a positive number $m$ such that each collection of mutually disjoint subcontinua of $X$ having diameters greater than $\varepsilon$ consists of at most $m$ elements [5, p. 132].

II.2. THEOREM. If there is a positive integer $n$ such that $f$ is an $n$-partially confluent map from a regular continuum $X$ onto a continuum $Y$, then $Y$ is regular.

Proof. Suppose $Y$ is not regular. Then there is a number $\varepsilon>0$ such that for each integer $m>0$ there is a collection of $m$ mutually disjoint continua in $Y$ each with diameter greater than $\varepsilon$.

By the continuity of $f$, there is a number $\delta>0$ such that if $C$ is a continuum in $X$ and $\operatorname{diam}(C)<\delta$ then $\operatorname{diam}(f(C))<\varepsilon / n$. By the $n$-partial confluence of $f$, for each continuum $K$ in $Y$ whose diameter is greater than $\varepsilon$ there is a continuum $K^{\prime}$ in $X$ such that $\operatorname{diam}\left(f\left(K^{\prime}\right)\right) \geq \varepsilon / n$. But, then $\operatorname{diam}\left(K^{\prime}\right) \geq \delta$. So, for each integer $m$ there is a collection of mutually disjoint continua in $X$ whose diameters are greater than $\delta$. This contradicts the fact that $X$ is regular.

II.3. THEOREM. If there is a positive integer $n$ such that $f$ is an $n$-partially confluent map from a finitely suslinean compactum $X$ onto a compactum $Y$, then $Y$ is finitely suslinean.

PROOF. The proof is almost identical to the proof of Theorem II.2. 
II.4. THEOREM. If $f$ is a partially confluent map from a hereditarily locally connected continuum $X$ onto a continuum $Y$, then $Y$ is hereditarily locally connected.

PROOF. Since continuous maps preserve local connectivity, each subcontinuum of $U$ is the finite union of locally connected continua and therefore is locally connected.

II.5. THEOREM. If $f$ is a partially confluent map from a hereditarily arcwise connected continuum $X$ onto a continuum $Y$, then $Y$ is hereditarily arcwise connected.

PROOF. The proof is similar to the proof of II.4.

The proof of the following theorem is almost the same as the proof of Theorem 5.5 of [6]. A continuum $X$ is acyclic if each map from $X$ onto a circle is homotopic to a constant map.

II.6. THEOREM. If $f$ is a partially confluent map from a one-dimensional acyclic continuum $X$ onto a continuum $Y$, then $Y$ is at most one-dimensional.

ProOF. Suppose the dimension of $Y$ is greater than one. Then there is a weakly confluent map $g$ from $Y$ onto a 2-cell $I^{2}$ [6, Theorem I, p. 328]. Clearly, $g \cdot f: X \rightarrow I^{2}$ is partially confluent. Let $D$ be a homeomorphic copy of

$$
\left\{(r, \theta) \mid r=1, r=2, \text { or } r=\left(2+e^{\theta}\right) /\left(1+e^{\theta}\right)\right\}
$$

in $I^{2}$. If $C$ is a subcontinuum of $Y$ such that $g \cdot f(C)$ is in $D$, the restriction of $g \cdot f$ to $C$ is homotopic to a constant map, so $g \cdot f(C)$ is contained in one of the three arc components of $D[3, \mathrm{p} .542]$. Since one of these arc components is not compact, $D$ is not the union of images under $g \cdot f$ of finitely many continua from $X$. This contradicts the partial confluence of $g \cdot f$.

Theorem II.7 is a generalization of Theorem 3 of [9] for weakly confluent maps. Again, the proof is almost the same.

II.7. THEOREM. If $f$ is a partially confluent map from a continuum $X$ onto a continuum $Y$ such that the dimension of $Y$ is greater than one, then $X$ contains uncountably many nonhomeomorphic subcontinua.

PROOF. There is a collection of continua in $I^{2}=[0,1] \times[0,1]$ called the Waraskiewicz spirals with the property that if $\Phi$ is a countable collection of continua there is a Waraskiewicz spiral $S$ such that no member of $\Phi$ maps onto $S$ [9, Theorem 2]. Each of these spirals is a ray limiting onto a circle.

As in the previous proof, let $g$ be a weakly confluent map from $Y$ onto $I^{2}$. If a finite collection of continua in $X$ has images under the partially confluent map $g \cdot f$ whose union is a particular Waraskiewicz spiral, then one of these continua must map onto the circle and part of the ray which spirals onto the circle, and thus one of these continua could be mapped onto all of the spirals. It follows that $X$ contains an uncountable collection of nonhomeomorphic continua.

An infinite-odd $X$ is a continuum which has a subcontinuum $Q$, called the core of $X$, such that $X \backslash Q$ has infinitely many components. A simple infinite-odd $X$ is an infinite-odd whose core is a point $p$ and such that each component of $X \backslash p$ is homeomorphic to the interval $(0,1]$. 
II.8. LEMMA. If $X$ is a suslinean continuum which does not contain an infiniteodd and $f$ is a partially confluent map from $X$ onto a continuum $Y$, then $Y$ does not contain a simple infinite-odd.

PROOF. This proof is similar to the proof of Theorem 5 of [4].

Suppose $Y$ contains the simple infinite-odd $F=A_{1} \cup A_{2} \cup A_{3} \cup \ldots$, where each $A_{i}$ is an arc and $p=A_{1} \cap A_{2} \cap A_{3} \cap \ldots$. Since $F$ is the union of the images of a finite number of continua which are images of continua in $Y$, one of these continua must map onto a simple infinite-odd contained in $F$. Therefore, with no loss of generality, it can be assumed that $Y=F$. Let $a_{i}$ refer to a homeomorphism from $[0,1]$ onto $A_{i}$ which sends 0 to $p$.

For each $\alpha$ in the interval $\left(\frac{1}{4}, 1\right)$ there is an infinite collection $I_{\alpha}$ of positive integers and a continuum $F_{\alpha}^{\prime}$ such that $f\left(F_{\alpha}^{\prime}\right)=\bigcup_{i \in I \alpha} a_{i}([0, \alpha])$. This follows from the partial confluence of $f$. For each $\alpha \in\left(\frac{1}{4}, 1\right)$ and $j \in I_{\alpha}$ there is a subcontinuum $K_{\alpha j}^{\prime}$ of $F_{\alpha}^{\prime}$ which maps onto $a_{j}\left(\left[\frac{1}{4}, \alpha\right]\right)$. This is because every continuum in $\bigcup_{i \in I_{\alpha}} a_{i}([0, \alpha])$ which contains $a_{j}(\alpha)$ and a point not in $a_{j}\left(\left[\frac{1}{4}, \alpha\right]\right)$ contains $a_{j}\left(\left[\frac{1}{4}, \alpha\right]\right)$, so $a_{j}\left(\left[\frac{1}{4}, \alpha\right]\right)$ is a $W$-set in $\bigcup_{i \in I \alpha} a_{i}([0, \alpha])$ by $[8$, Lemma 2 , p. 165].

For each $\varepsilon$ in $\left(\frac{1}{4}, 1\right)$ it is not the case that for each $j \in I_{\varepsilon}$ there is an $\alpha \in(\varepsilon, 1)$ such that $j \in I_{\alpha}$ and $F_{\varepsilon}^{\prime} \cap K_{\alpha j}^{\prime} \neq \varnothing$ or else $F_{\varepsilon}^{\prime}$ is the core of an infinite-odd in $X$. So for each $\varepsilon$ in $\left(\frac{1}{4}, 1\right)$ there is a $j(\varepsilon)$ in $I_{\varepsilon}$ such that if $\delta$ is in $(\varepsilon, 1]$, and $j(\varepsilon)$ is in $I_{\delta}$, then $F_{\varepsilon}^{\prime} \cap K_{\delta j(\varepsilon)}^{\prime}$ is empty. But then there is an uncountable set $E$ in $\left(\frac{1}{4}, 1\right)$ such that if $\varepsilon$ and $\delta$ are in $E$ then $j(\varepsilon)=j(\delta)$.

If $\varepsilon$ and $\gamma$ are in $E$ then $K_{\varepsilon j(\varepsilon)}^{\prime} \subset F_{\varepsilon}^{\prime}$ and $F_{\varepsilon j(\varepsilon)}^{\prime} \cap K_{\gamma j(\varepsilon)}=\varnothing$. But $j(\varepsilon)=j(\gamma)$ so $K_{\varepsilon j(\varepsilon)}^{\prime} \cap K_{\gamma j(\gamma)}^{\prime}=\varnothing$. Therefore, $\left\{K_{\varepsilon j(\varepsilon)}^{\prime} \mid \varepsilon\right.$ is in $\left.E\right\}$ is an uncountable collection of pairwise disjoint nondegenerate continua in $X$; this contradicts the fact that $X$ is suslinean.

Note that Example I.2 demonstrates that partial confluence cannot be replaced by pseudoconfluence in the hypothesis of the preceding theorem.

In [2, Theorem 2.4, p. 41] Cook and Lelek prove that if a continuum $X$ contains a triod then there is a weakly confluent map from $X$ onto a simple triod. The same argument, with slight variation, will show that if $F$ is the simple $n$-odd

$$
F=\{(r, \theta) \mid \theta=1 / n \text { and } 0 \leq r \leq \theta \text { for some } n=1,2,3, \ldots\},
$$

and $X$ is a continuum which contains an infinite-odd, then there is a weakly confluent map from $X$ onto $F$.

II.9. THEOREM. If $X$ is a suslinean continuum which does not contain an infinite-odd and $f$ is a partially confluent map from $X$ onto a continuum $Y$, then $Y$ does not contain an infinite-odd.

PROOF. If $Y$ contains an infinite-odd then there is a weakly confluent map $g$ from $Y$ onto the simple infinite-odd $F$ above. The map $g \cdot f$ is partially confluent, contradicting Lemma II.8.

II.10. THEOREM. A continuum $Y$ is suslinean and does not contain an infiniteodd if and only if there does not exist a partially confluent map of $Y$ onto $F$.

PROOF. Theorem II.9 states the necessity of the condition. On the other hand, if $Y$ is not suslinean there is a weakly confluent map from $Y$ onto $F[\mathbf{2}$, Theorem 
2.3, p. 41]. If $Y$ contains an infinite-odd, it was noted above that there is a weakly confluent map from $Y$ onto $F$.

III. Partially confluent maps and graphs. In this section it is shown that not only is the partially confluent image of a graph a graph, but that partially confluent maps are the most general class of maps which map graphs onto graphs. In fact, it is shown that each map from a continuum onto a graph is partially confluent.

A graph is a continuum which is a finite union of arcs called edges which intersect only at their endpoints. The following is a well-known characterization of graphs [10, p. 182]:

A continuum $X$ is a graph if and only if all but a finite number of the points of $X$ have order two.

III.1. THEOREM. A continuum is a graph if and only if it is hereditarily locally connected and does not contain an infinite-odd.

ProOF. One direction is obvious.

Assume $X$ is a hereditarily locally connected continuum which does not contain an infinite-odd. In order to get a contradiction, assume $X$ is not a graph. Two cases arise.

In the first case, $X$ has a finite number of branch points, and, in this case $X$ must have an infinite number of endpoints. If $e$ is an endpoint, then there is an $\operatorname{arc} A$ in $X$ from $e$ to a branch point. Since the $\operatorname{arc} A$ contains at most a finite number of branch points, there is an $\operatorname{arc} A^{\prime}$ from $e$ to a branch point in $X$ such that $A^{\prime}$ contains no other branch points. Note that the choice of such an arc for each endpoint is unique. Now, since there are infinitely many endpoints and only a finite number of branch points, one of the branch points is the core of an infinite-odd. This is a contradiction.

In the second case, $X$ has an infinite number of branch points. Let $F_{0}$ be a set consisting of a single branch point $x_{0}$. There is an arc $A$ from $x_{0}$ to another branch point. Such an arc can contain at most a finite number of branch points or else the arc would be the core of an infinite-odd. So there is a branch point $y$ in $A$ not equal to $x_{0}$ and an arc from $x_{0}$ to $y$ such that no point on that arc other than its endpoints is a branch point. Let $F_{1}$ be the set of all branch points $y$ not in $F_{0}$ such that there is an arc from $x_{0}$ to $y$ such that no point of the arc is a branch point except its endpoints. The set $F_{1}$ must be finite or else $x_{0}$ would be the core of an infinite-odd. Let $F_{2}$ be the set of all branch points $y$ not in $F_{0} \cup F_{1}$ such that there is an arc from a point in $F_{1}$ to $y$ such that no point of the arc is a branch point except its endpoints. Again, $F_{2}$ must be finite or some point in $F_{1}$ is the core of an infinite-odd.

Since there are infinitely many branch points, this process does not end, and there is an infinite sequence $x_{0}, x_{1}, x_{2}, \ldots$ of points such that each $x_{i}$ is in $F_{i}$ and such that for each $i$ there is an arc $A_{i}$ from $x_{i-1}$ to $x_{i}$ such that no point of the arc is a branch point except its endpoints. Let $K$ be the closure of the union of the $A_{i}$ 's. Since $K$ is locally connected, it is either an arc, a simple closed curve, or the union of a simple closed curve and an arc which intersects the simple closed curve only at one endpoint. In any case, $K$ is the core of an infinite-odd since $K$ contains infinitely many branch points, and this is a contradiction. 
III.2. THEOREM. If $f$ is a partially confluent map from a graph $X$ onto a continuum $Y$, then $Y$ is a graph.

Proof. By Theorem II.4, $Y$ is hereditarily locally connected, and by Theorem II.8, $Y$ does not contain an infinite-odd. So, by Theorem III.1, $Y$ is a graph.

III.3. THEOREM. If $f$ is a map from a continuum onto a graph $Y$, then $f$ is partially confluent.

ProOF. Let $A_{1}, A_{2}, \ldots, A_{n}$ be the edges of $Y$. For each $i$, there are components of $f^{-1}\left(A_{i}\right)$ whose images are maximal with respect to containing one or the other of the two endpoints of $A_{i}$, and $A_{i}$ is the union of two or fewer of the images under $f$ of such components of $f^{-1}\left(A_{i}\right)$. If $C$ is a subcontinuum of $A_{i}$ for some $i$, then $C$ is the union of two or fewer of the images under $f$ of the components of $f^{-1}(C)$. If $K$ is a subcontinuum of $Y$, then $K$ is the union of a finite number of its subcontinua $K_{1}, K_{2}, \ldots, K_{m}$, each contained in an $A_{i}$ for some $i$. Since each $K_{i}$ is the union of two or fewer of the images under $f$ of the components of $f^{-1}\left(K_{i}\right), K$ is the union of a finite number of images under $f$ of components of $f^{-1}(K)$.

The previous two theorems combine for the following.

III.4. THEOREM. If $f$ is a map from a graph $X$ onto a continuum $Y$, then $Y$ is a graph if and only if $f$ is partially confluent.

IV. Continua which are the images of partially confluent maps only. The class of continua which are the images of weakly confluent maps only, class $[W]$, has been studied and shown to contain all chainable continua, among other types of continua. But, class $[W]$ certainly does not contain a simple triod. In the previous section it was shown that graphs are the images of partially confluent maps only. In this section it is shown that the inverse limit on a fixed acyclic graph is the image of partially confluent maps only.

A map $f$ from a continuum $X$ onto a continuum $Y$ is said to be weakly confluent with respect to a subcontinuum $K$ of $Y$ if some component of $f^{-1}(K)$ maps onto $K$. A subcontinuum $K$ of a continuum $Y$ is called a $W$-set in $Y$ if each map from a continuum onto $Y$ is weakly confluent with respect to $K$.

IV.1. THEOREM. Suppose $G$ is an acyclic graph with exactly $n$ edges, and $X$ is an inverse limit of $G$. Then each subcontinuum of $X$ is the union of $n$ or fewer $w$-sets.

Proof. Let $K$ be a subcontinuum of $M$. Since each subcontinuum of an edge of $G$ is a $w$-set in $G$ [8, Lemma 3, p. 165], the projection $\pi_{i} \mid M \rightarrow G$ is weakly confluent with respect to $\pi_{i}(K) \cap E_{i}$ for each of the edges $E_{1}, E_{2}, E_{3}, \ldots, E_{n}$ of $G$. For each positive integer $i$ and integer $j$ from 1 to $n$ let $K_{j i}$ be a subcontinuum of $K$ which projects onto $\pi_{i}(K) \cap E_{j}$. There is a subsequence $u$ of the positive integers such that $\left\{K_{j u(i)}\right\}$ converges to a subcontinuum $K_{j}$ of $K$ for each $j$. The union of the $K_{j}$ 's is $K$, since if $x$ is in $K$, then for each $i$ there is a $j$ and a point $x_{j i} \in K_{j u(i)}$ such that $\pi_{u(i)}\left(x_{j i}\right)=\pi_{u(i)}(x)$, and therefore, the sequence $\left\{x_{j i}\right\}$ converges to $x$, so $x \in U_{j=1 \ldots n} K_{j}$.

The $K_{j}$ 's which are not empty are $w$-sets in $M$. To see this, let $f$ be a map from a continuum $X$ onto $Y$. For each positive integer $i$ and integer $j$ from 1 to $n, \pi_{u(i)} \circ f$ is weakly confluent with respect to $\pi_{u(i)}(K) \cap E_{j}$. Let $C_{j i}$ be a subcontinuum of $N$ 
such that $f\left(C_{j i}\right)=\pi_{u(i)}(K) \cap E_{i}$. There is a subsequence $v$ of $u$ such that $\left\{C_{j i}\right\}$ converges to a continuum $C_{j}$ in $Y$ for each $j$. Let $x$ be an element of $K_{j}$, and let $\left\{x_{j i}\right\}$ be a sequence of points converging to $x$ such that $x_{j i} \in K_{j v(i)}$. For each $i$ there is a point $c_{j i}$ in $C_{j v(i)}$ such that $\pi_{v(i)}\left(f\left(c_{j i}\right)\right)=\pi_{v(i)}\left(x_{j i}\right)$. The sequence $\left\{f\left(c_{j i}\right)\right\}$ converges to $x$, so some subsequence of $\left\{c_{j i}\right\}$ converges to a point $c$ in $C_{j}$ and $f(c)=x$. Therefore, $K_{j} \subset f\left(C_{j}\right)$. On the other hand, if $f(c)$ is in $f\left(C_{j}\right)$ then there is a sequence $\left\{c_{j i}\right\}$ converging to $c$ such that $c_{j i} \in C_{j v(i)}$. But, then there is a sequence $\left\{x_{j i}\right\}$ of points such that $x_{j i} \in K_{j i}$ and $\pi_{v(i)}\left(x_{j i}\right)=\pi_{v(i)}\left(f\left(c_{j i}\right)\right)$. Some subsequence of $\left\{x_{j i}\right\}$ converges to a point $x$ in $K_{j}$, and the sequence $\left\{f\left(c_{j i}\right)\right\}$ converges to $x$. Since $f(c)=x, f(c)$ is in $K_{j}$. Therefore, $f\left(C_{j}\right) \subset K_{j}$.

So, each map from a continuum onto $X$ is weakly confluent with respect to $K_{j}$ whenever $K_{j}$ is nonempty and, consequently, $K$ is the union of $n$ or fewer $W$-sets.

IV.2. COROLlaRY. Suppose $G$ is an acyclic graph with exactly $n$ edges, $X$ is an inverse limit of $G$, and $f$ is a map from a continuum onto $X$; then $f$ is $n$-partially confluent.

V. Remarks. It follows from Corollary IV.2 that an acyclic graph $G$ is the image of $n$-partially confluent maps only where $n$ is the number of edges of $G$. A closer look at the proof of Theorem III.3 reveals that any graph $G$ is the image of $n$-partially confluent maps only where $n$ is definitely smaller than four times the number of edges of $G$.

VI. Question. Is every map from a continuum onto the inverse limit of a graph partially confluent?

The author would like to thank Jim Davis for the suggestion that Theorem III.4 might be true.

\section{REFERENCES}

1. C. A. Eberhart, J. B. Fugate, and G. R. Gordh, Jr., Branchpoint covering theorems for confluent and weakly confluent maps, Proc. Amer. Math. Soc. 55 (1976), 409-415.

2. H. Cook and A. Lelek, Weakly confluent mappings and atriodic suslinean curves, Canad. J. Math. 30 (1978), 32-44.

3. M. K. Fort, Jr., Images of plane continua, Amer. J. Math. 81 (1959), 541-546.

4. E. E. Grace and E. J. Vought, Semi-confluent and weakly confluent images of tree-like and atriodic continua, Fund. Math. 101 (1978), 151-158.

5. A. Lelek, On topology of curves. II, Fund. Math. 70 (1971), 131-138.

6. A. Lelek and E. D. Tymchatyn, Pseudo-confluent mappings and a classification of continua, Canad. J. Math. 27 (1975), 1336-1348.

7. S. Mazurkiewicz, Sur l'existence des continues indecomposables, Fund. Math. 25 (1935), 327328.

8. V. C. Nall, Weak confluence and W-sets, Topology Proc. 8 (1983), 161-193.

9. J. T. Rogers, Orbits of higher-dimensional hereditarily indecomposable continua, Proc. Amer. Math Soc. (to appear).

10. G. T. Whyburn, Analytic topology, Amer. Math. Soc. Colloq. Publ., vol. 28, Amer. Math. Soc., Providence, R.I., 1963.

Department of Mathematics, University of Richmond, Richmond, Virginia 23173 